\title{
Comparison and diagnosis of Entamoeba in stool sample from rural community of Nepal
}

\section{Sandeep Thapa}

Microbial Genetics, Kathmandu Center for Genomics and Research Laboratory, Gwarko, State 2: Lalitpur, Nepal

Objective

To find out the prevalence of Entamoeba species in rural community of Nepal.

The purpose of the study is to evaluate Nested PCR, microscopic examination and Elisa assay for detection and differentiation of Entamoeba species.

\section{Introduction}

Nepal being a developing country has many health problems, which escalate in intensity at different times of the year or in epidemic form. Amebiasis is one of the infectious diseases that is highly seen in rural area of Nepal caused by Entamoeba species [1,2]. Recent reports show that open defecation, drinking untreated water, unsanitary habits and lack of basic health knowledge cause higher mortality and morbidity in our country.

E. histolytica is an anaerobic pathogenic parasitic. However, E. dispar and E.moshkovskii exits as non-pathogenic. Likewise, E. histolytica, E. dispar and E. moshkovskii are morphologically identical but genetically distinct species [3].

\section{Methods}

A total of 270 faecal sample were collected from south eastern terai region of Nepal after the informed consent form. The samples were processed by direct wet smear and formalin ethyl acetate concentration technique [4]. Eventually, microscopic examination were performed for the detection of Entamoeba species along with other intestinal parasites. Furthermore, enzyme immunoassay were executed to detect antigens of E. histolytica through ELISA. Additionally, microscopically positive samples for Entamoeba species cysts were further characterized using a Nested- PCR targeting 16S-like ribosomal RNA gene [5]. The PCR generate amplicons which was subjected to $2 \%$ agarose gels electrophoresis and visualized under UV transilluminator.

\section{Results}

$8.52 \%$ of the total collected samples were microscopically positive for Entamoeba cysts either singly or in combination with other intestinal parasites. Likewise, among 270 stool sample, viral diarrheal was most significant form of diarrhoea found in $76.67 \%$ of patients. Among different organisms, As. Lumbricoids and E. histolytica, G. lambia and H. nana were identified in most of the patients accounting for $11.11 \%, 8.52 \%, 2.59 \%$ and $1.11 \%$ respectively. However, Lumbricoids, G. lambia, Tenia solium and E. histolytica were present in an individual patient while two patient was found with both As. Lumbricoids and G. lambia. Among several symptoms, diarrhoea seems to be the common symptoms infecting all of the patients which is followed by fever and vomiting which accounts for $55.1 \%$ and $46.2 \%$ correspondingly. Whereas, nausea appears to be the least common symptoms infecting only $14.4 \%$ of patients.

Subsequently, 56 cases were PCR positive, 51 cases were ELISA positive whereas 47 were found to be positive by microscopy.

\section{Conclusions}

Molecular techniques are indeed promising tools for epidemiological studies, particularly in discriminating the pathogenic from the non-pathogenic species of the Entamoeba species. This study reports a new nested multiplex PCR strategy for detection and differentiation of E. histolytica, E. dispar and E. moshkovskii which is highly rapid, specific and sensitive which is useful for proper diagnosis, immunological assay and drug testing. 
ISDS 2019 Conference Abstracts

\section{Acknowledgement}

This work was supported by the Wellcome TDR-WHO. We would like to thank all the member of KCGRL and Global Hospital.

\section{References}

1. Chaudhary M, Maharjan M. 2014. Association of Anaemia with Parasitic Infection in Pregnant Women Attending Antenatal Clinic at Koshi Zonal Hospital. Neplalese Journal of Zoology. 2(1), 1-7.

2. Tandukar S, Ansari S, Adhikari N, Shrestha A, Gautam J, et al. 2013. Intestinal parasitosis in school children of Lalitpur district of Nepal. BMC Res Notes. 6(1), 449. PubMed https://doi.org/10.1186/1756-0500-6-449

3. Clark C, Espinosa Cantellano M, Bhattacharya A. Entamoeba histolytica: an overview of the biology of the organism (2013). Amebiasis: World Scientific, 1-45.

4. Dhanabal J, Selvadoss PP, Muthuswamy K. 2014. Comparative study of the prevalence of intestinal parasites in low socioeconomic areas from South Chennai, India. J Parasitol Res. 2014, 630968. PubMed https://doi.org/10.1155/2014/630968

5. Van den Bossche D, Cnops L, Verschueren J, Van Esbroeck M. 2015. Comparison of four rapid diagnostic tests, ELISA, microscopy and PCR for the detection of Giardia lamblia, Cryptosporidium spp. and Entamoeba histolytica in feces. J Microbiol Methods. 110, 78-84. PubMed https://doi.org/10.1016/j.mimet.2015.01.016 